\title{
Cell-cell adhesion through N-cadherin enhances VCAM-1 expression via PDGFR $\beta$ in a ligand-independent manner in mesenchymal stem cells
}

\author{
EMIKO AOMATSU $^{1,2^{*}}$, NAOYUKI CHOSA ${ }^{1 *}$, SOKO NISHIHIRA $^{1,3}$, \\ YOSHIKI SUGIYAMA ${ }^{3}$, HIROYUKI MIURA ${ }^{2}$ and AKIRA ISHISAKI ${ }^{1}$ \\ ${ }^{1}$ Division of Cellular Biosignal Sciences, Department of Biochemistry, Iwate Medical University, Yahaba, \\ Iwate 028-3694; ${ }^{2}$ Division of Orthodontics, Department of Developmental Oral Health Science, \\ ${ }^{3}$ Division of Oral Surgery, Department of Oral and Maxillofacial Surgery, Iwate Medical University \\ School of Dentistry, Morioka, Iwate 020-8505, Japan
}

Received November 19, 2013; Accepted December 17, 2013

DOI: 10.3892/ijmm.2013.1607

\begin{abstract}
Cell-cell adhesions induce various intracellular signals through hierarchical and synergistic molecular interactions. Recently, we demonstrated that a high cell density induces the expression of vascular cell adhesion molecule-1 (VCAM-1) through the nuclear factor- $\kappa \mathrm{B}(\mathrm{NF}-\kappa \mathrm{B})$ pathway in human bone marrow-derived mesenchymal stem cells (MSCs). However, the specific molecules that activated the $\mathrm{NF}-\kappa \mathrm{B}$ pathway were not determined. In the present study, in experiments with receptor tyrosine kinase inhibitors, VCAM-1 expression was completely suppressed by platelet-derived growth factor (PDGF) receptor (PDGFR) inhibitors. In addition, VCAM-1 expression was significantly suppressed by knockdown with PDGFR $\beta$ siRNA, but not with PDGFR $\alpha$ siRNA. However, VCAM-1 expression did not increase following treatment with PDGF. The overexpression of $\mathrm{N}$-cadherin, a structural molecule in adherence junctions in MSCs, promoted VCAM-1 expression and induced the marked phosphorylation of the intracellular signaling factor, Src. In addition, VCAM-1 expression and Src phosphorylation were reduced by the overexpression of a dominant negative mutant of $\mathrm{N}$-cadherin. These results suggest that cell-cell adhesion, through $\mathrm{N}$-cadherin, enhances the expression of VCAM-1 via PDGFR $\beta$ and the activation of Src in a ligand-independent manner in MSCs.
\end{abstract}

Correspondence to: Dr Naoyuki Chosa, Division of Cellular Biosignal Sciences, Department of Biochemistry, Iwate Medical University, 2-1-1 Nishitokuta, Yahaba, Iwate 028-3694, Japan

E-mail: nchosa@iwate-med.ac.jp

*Contributed equally

Key words: mesenchymal stem cells, cell-cell adhesion, vascular cell adhesion molecule-1, N-cadherin, platelet-derived growth factor receptor $\beta$, Src

\section{Introduction}

Mesenchymal stem cells (MSCs) are non-hematopoietic stromal cells, which retain the ability to self-renew and differentiate into mesenchymal cells, such as osteoblasts, adipocytes, chondrocytes and skeletal muscle cells (1). Various surface markers, including CD49a, CD73, CD105, vascular cell adhesion molecule-1 (VCAM-1)/CD106, CD140b, CD146, CD271, mesenchymal stromal cell antigen-1 (MSCA-1) and STRO-1, have been used alone or in combination to identify and isolate human MSCs (hMSCs) (2-8). STRO-1 is a popular MSC marker and is often used in combination with VCAM-1 for MSC isolation. A number of studies have analyzed the changes in surface marker expression caused by prolonged cultivation, and have reported the attenuated expression of several markers, including VCAM-1 (9,10). Cell adhesion molecules, such as VCAM-1 mediate the interaction of MSCs with endothelial cells (ECs), which is essential for MSC homing. Therefore, the reduction in VCAM-1 expression during expansion in culture may be related to the decreased homing ability of senescent MSCs. Mabuchi et al recently demonstrated that clones of hMSCs retaining high rates of colony-forming unit fibroblasts (CFU-Fs) expressing the surface markers, CD271/ low affinity nerve growth factor receptor (LNGFR), thymocyte antigen-1 (Thy-1) and VCAM-1, exhibited robust multilineage differentiation and self-renewal ability (11). An in-depth investigation of MSC markers has made it possible to identify and purify MSCs; for instance, anti-CD49a antibody is useful for identifying hMSCs $(12,13)$. Notably, rapidly expanding clones of hMSCs express high levels of CD49a and VCAM-1 and are highly migratory (11). These results suggest that VCAM-1 can be used as a marker for enriching migratory, multipotent and proliferative cells from culture-expanded MSCs.

Cadherins are members of a family of transmembrane proteins involved in mediating homophilic adhesion in a $\mathrm{Ca}^{2+}$-dependent manner. These proteins are major components of adherence junctions (AJs) in cells. E-cadherin is the main cadherin in the AJs of epithelial cells, whereas other cadherins, including N-cadherin, P-cadherin, R-cadherin and 
VE-cadherin form AJs in other cell types. Although fibroblasts express a number of different cadherins, including P-cadherin, R-cadherin, OB-cadherin and fat-like cadherins $(14,15)$, $\mathrm{N}$-cadherin is the predominant cadherin expressed by these cells $(14,16)$. N-cadherin-mediated AJs are of great importance in connective tissue physiology and are critical for the regulation of cell attachment and migration (17), wound healing (18), metastatic potential (19) and embryonic development $(20,21)$, as well as the differentiation and formation of numerous specialized tissues, including fibrous connective tissues (22-26). N-cadherin is considered to be the key factor in directing cell-cell interactions during mesenchymal condensation, a process mediated by surface contact that results in the aggregation of progenitor cells (27-30). Studies have indicated that the expression of deletion mutant forms of N-cadherin, which lack either the extracellular homotypic interaction domains or the intracellular $\beta$-catenin binding site, results in decreased cellular condensation and impaired chondrogenesis $(31,32)$. These findings suggest that both extracellular homotypic interaction and intracellular interaction with the catenin complex are essential for proper $\mathrm{N}$-cadherin signaling (33).

In a recent study of ours, we found that the expression level of VCAM-1/CD106 was markedly upregulated in human bone marrow-derived MSCs, UE7T-13 cells, under conditions of high cell density (34). The high cell density-induced expression of VCAM-1 was markedly suppressed by nuclear factor- $\kappa \mathrm{B}$ $(\mathrm{NF}-\kappa \mathrm{B})$ signaling-related protein kinase inhibitors, such as the I $\kappa$ B kinase-2 (IKK-2) inhibitor VI, a phosphoinositide 3-kinase (PI3K) inhibitor, an Src inhibitor and a protein kinase $\mathrm{C}$ (PKC) inhibitor. Therefore, the high cell densityinduced VCAM-1 expression was regulated by the $\mathrm{NF}-\kappa \mathrm{B}$ pathway in human bone marrow-derived MSCs. However, the identities of the inducing factor(s) that activate the $\mathrm{NF}-\kappa \mathrm{B}$ pathway in MSC cell-cell adhesion are not yet clear. Herein, we demonstrate that cell-cell adhesion by N-cadherin activates the NF- $\kappa$ B pathway via platelet-derived growth factor (PDGF) receptor (PDGFR) $\beta$ in a ligand-independent manner.

\section{Materials and methods}

Reagents. The SCADS inhibitor kit, including various protein kinase inhibitors was generously supplied by the Screening Committee of Anticancer Drugs supported by a Grantin-Aid for Scientific Research on Priority Area 'Cancer' from the Ministry of Education, Culture, Sports, Science and Technology, Japan.

Cell culture. Human bone marrow-derived MSCs, UE7T-13 cells, the life span of which was prolonged by infection with a retrovirus encoding human papillomavirus E7 and human telomerase reverse transcriptase (hTERT) $(35,36)$, were purchased from the Health Science Research Resources Bank (JCRB no. 1154, Japan Health Sciences Foundation, Japan). UE7T-13 cells were cultured in Dulbecco's modified Eagle's medium (DMEM; Sigma-Aldrich, St. Louis, MO, USA) supplemented with $10 \%$ fetal bovine serum (FBS; HyClone, Logan, UT, USA) at $37^{\circ} \mathrm{C}$ in a humidified incubator with an atmosphere of $5 \% \mathrm{CO}_{2}\left(3.0 \times 10^{3}\right.$ cells $/ \mathrm{cm}^{2}$, 'low' cell density; $1.0 \times 10^{5}$ cells $/ \mathrm{cm}^{2}$, 'high' cell density).
RNA isolation and quantitative RT-PCR ( $q R T-P C R)$. Total RNA from low, medium and high cell density cultured UE7T-13 cells was isolated using Isogen reagent (Nippon Gene, Tokyo, Japan) according to the manufacturer's instructions. Firststrand cDNA was synthesized from total RNA by using the PrimeScript RT reagent kit (Takara, Kyoto, Japan). qRT-PCR was performed on a Thermal Cycler Dice Real-time System (Takara) using SYBR Premix Ex Taq II (Takara) with specific oligonucleotide primers (presented in Table I). The mRNA expression levels for VCAM-1, PDGFR $\alpha$ and PDGFR $\beta$ were normalized to those obtained for glyceraldehyde adenosinephosphate dehydrogenase $(\mathrm{GAPDH})$, and the relative expression levels were shown as fold-increase or decrease relative to the control.

Western blot analysis. The UE7T-13 cells were washed twice with ice-cold PBS and then lysed in RIPA buffer (50 mM Tris- $\mathrm{HCl}, \mathrm{pH} 7.2,150 \mathrm{mM} \mathrm{NaCl}, 1 \% \mathrm{NP}-40,0.5 \%$ sodium deoxycholate and $0.1 \%$ SDS) containing protease and phosphatase inhibitor cocktails (Sigma-Aldrich). The protein content of the samples was measured using the BCA reagent (Pierce Biotechnology, Rockford, IL, USA). Samples containing equal amounts of protein were separated by $12.5 \%$ SDS-polyacrylamide gel electrophoresis (SDS-PAGE) and transferred onto a polyvinylidene difluoride (PVDF) membrane (Millipore, Billerica, MA, USA). After being blocked with 5\% non-fat dry milk in T-TBS (50 mM Tris- $\mathrm{HCl}$, $\mathrm{pH} 7.2,150 \mathrm{mM} \mathrm{NaCl}$ and $0.1 \%$ Tween-20), the membrane was incubated with primary anti-Src (Cell Signaling, Technologies, Beverly, MA, USA) and anti-phospho-Src (p-Src, Cell Signaling) antibodies, using an anti- $\beta$-actin (clone C4, Santa Cruz Biotechnology Inc., Santa Cruz, CA, USA) antibody as a loading control for normalization. The blots were subsequently incubated with horseradish peroxidase-conjugated secondary antibody and developed using chemiluminescence with the Amersham ECL western blot analysis system (GE Healthcare Biosciences, Pittsburgh, PA, USA). The detected blots were photographed using the photo image detection system CL-Cube L (Tohoku Electronic Industrial Co., Ltd., Sendai, Japan) and densitometrically measured using ImageJ software (version 1.44). Data are expressed as the ratio of phosphorylated to total molecular bands.

siRNA transfection for PDGFRs. Three sets of Stealth siRNA oligonucleotide duplexes targeting PDGFR $\alpha$ and PDGFR $\beta$ were designed using the online BLOCK-iT RNAi Designer software (Invitrogen, Carlsbad, CA, USA). Sequences of the siRNA oligonucleotide duplexes are listed in Table II. UE7T-13 cells were seeded in 24-well culture plates without antibiotic selection at a density of $2.0 \times 10^{4}$ cells/well, $24 \mathrm{~h}$ before siRNA transfection. Subsequently, transcriptional knockdown was performed by transfection of the cells with siRNA oligonucleotide duplexes at a final concentration of $20 \mathrm{nM}$ in DMEM using Lipofectamine RNAiMAX (Invitrogen) for $24 \mathrm{~h}$ according to the manufacturer's instructions. The effects of RNAi knockdown of target genes were assayed by qRT-PCR. Stealth siRNA Negative Control medium GC Duplex (Invitrogen) was also included as a control for sequence independent RNAi knockdown. 
Table I. Sequences of the qRT-PCR primers used in this study.

\begin{tabular}{|c|c|c|c|}
\hline Gene & Primer sequences & Location (bp) & Product size (bp) \\
\hline VCAM-1 & $\begin{array}{l}\text { Foward: 5'-CGAAAGGCCCAGTTGAAGGA-3' } \\
\text { Reverse: 5'-GAGCACGAGAAGCTCAGGAGAAA-3' }\end{array}$ & $2086-2226$ & 141 \\
\hline PDGFR $\alpha$ & $\begin{array}{l}\text { Forward: 5'-GTGCGAAGACTGAGCCAGATTG-3' } \\
\text { Reverse: 5'-CGATAAACAGAATGCTTGAGCTGTG-3' }\end{array}$ & $5708-5828$ & 121 \\
\hline PDGFR $\beta$ & $\begin{array}{l}\text { Forward: 5'-TGCCTTGCCAGCACTAACATTC-3' } \\
\text { Reverse: 5'-CCAGAGTGTGATGTGTGATCTGGA-3' }\end{array}$ & 4769-4908 & 140 \\
\hline GAPDH & $\begin{array}{l}\text { Forward: 5'-GCACCGTCAAGGCTGAGAAC-3' } \\
\text { Reverse: 5'-ATGGTGGTGAAGACGCCAGT-3' }\end{array}$ & $248-389$ & 142 \\
\hline
\end{tabular}

VCAM-1, vascular cell adhesion molecule-1; PDGFR $\alpha$, platelet-derived growth factor receptor $\alpha$; PDGFR $\beta$, platelet-derived growth factor receptor $\beta$; GAPDH, glyceraldehyde adenosine-phosphate dehydrogenase.

Table II. Oligonucleotide sequences of the Stealth siRNA duplex.

\begin{tabular}{|c|c|c|c|}
\hline Gene & siRNA no. & & RNA duplex sequence \\
\hline \multirow[t]{6}{*}{ PDGFR $\alpha$} & -387 & Sense: & 5'-AAUGAAAGCUGGCAGAGGAUUAGGC-3' \\
\hline & & Antisense: & 5'-GCCUAAUCCUCUGCCAGCUUUCAUU-3' \\
\hline & -751 & Sense: & 5'-UAUAAUGGCAGAAUCAUCAUCCUCC-3' \\
\hline & & Antisense: & 5'-GGAGGAUGAUGAUUCUGCCAUUAUA-3' \\
\hline & -843 & Sense: & 5'-UUAAAGCCCUGUCUGCUGUCGUAGG-3' \\
\hline & & Antisense: & 5'-CCUACGACAGCAGACAGGGCUUUAA-3' \\
\hline \multirow[t]{6}{*}{ PDGFR $\beta$} & -803 & Sense: & 5'-CAAAGAUGUAGAGCCGUUUCCGCUC-3' \\
\hline & & Antisense: & 5'-GAGCGGAAACGGCUCUACAUCUUUG-3' \\
\hline & -1061 & Sense: & 5'-CAUAGUAGGCAUCAGAAUCCACCUC-3' \\
\hline & & Antisense: & 5'-GAGGUGGAUUCUGAUGCCUACUAUG-3' \\
\hline & -1506 & Sense: & 5'-UUGUCUUUGAACCACAGGACAGUGG-3' \\
\hline & & Antisense: & 5'-CCACUGUCCUGUGGUUCAAAGACAA-3' \\
\hline
\end{tabular}

PDGFR $\alpha$, platelet-derived growth factor receptor $\alpha$; PDGFR $\beta$, platelet-derived growth factor receptor $\beta$.

Overexpression of $N$-cadherin. cDNA encoding full-length $\mathrm{N}$-cadherin $\left(\mathrm{Met}^{1}-\mathrm{Asp}^{906}\right)$ or an $\mathrm{N}$-cadherin deletion mutant lacking the C-terminal intercellular domain $\left(\operatorname{Met}^{1}-\operatorname{Trp}^{745}\right)$ was amplified by PCR. The cDNA was then subcloned into the pcDNA/V5/GW/D-TOPO vector (Invitrogen) using the pcDNA Gateway Directional TOPO Expression kit (Invitrogen) according to the manufacturer's instructions. The overexpression vectors, containing full-length $\mathrm{N}$-cadherin (pCDH2-full) or the deletion mutant $(\mathrm{pCDH} 2-\Delta)$, were transfected into the UE7T-13 cells using Lipofectamine LTX (Invitrogen) for $48 \mathrm{~h}$ according to the manufacturer's instructions. The effects of N-cadherin overexpression were assayed by flow cytometric analysis. The transfected UE7T-13 cells were stripped with cell dissociation buffer (Invitrogen) and washed with PBS containing 0.5\% FBS and 2 mM EDTA. The cells $\left(1.0 \times 10^{5}\right)$ were then incubated with an anti-N-cadherin antibody (clone GC-4; Abcam, Cambridge, MA, USA) for $1 \mathrm{~h}$ at room temperature. The cells were then incubated with phycoerythrin-conjugated secondary antibody for $1 \mathrm{~h}$. Image acquisition was performed using the EPICS XL ADC System (Beckman Coulter, Brea, CA, USA).
Cell proliferation assay. Cell proliferation was analyzed using a colorimetric assay for the quantification of the cleavage of the tetrazolium salt WST-1 (Roche Applied Science, Basel, Switzerland) by mitochondrial dehydrogenases in viable cells. The dye formed can be quantified using a spectrophotometer and directly correlates with the number of metabolically active cells in the culture. The cells were grown in 96-well plates (Nunclon ${ }^{\circledR}$; Sigma-Aldrich) for 1, 3 and 5 days treated with or without $10 \mathrm{ng} / \mathrm{ml}$ of recombinant human PDGF-BB (Acris Antibodies, San Diego, CA, USA). After each incubation period, the cells were incubated for a further $1 \mathrm{~h}$ at $37^{\circ} \mathrm{C}$ with $100 \mu \mathrm{l}$ medium containing $10 \mu \mathrm{l}$ WST-1 reagent. The samples were shaken for $1 \mathrm{~min}$, and absorbance was measured at $450 \mathrm{~nm}$ using a MPR-A4i microplate reader (Tosoh Corp., Tokyo, Japan).

Statistical analysis. All experiments were repeated at least 3 times. Representative images or data are shown. Data are presented as the means \pm standard deviation (SD). Differences between averages and percentages between the control and test samples were statistically analyzed using paired two-tailed 
Student's t-tests. Values of $\mathrm{p}<0.05$ were considered to indicate statistically significant differences.

\section{Results}

Effect of receptor tyrosine kinase (RTK) inhibitors on high cell density-induced VCAM-1 expression. In a recent study, we demonstrated that high cell density induces VCAM-1 expression through the NF- $\mathrm{KB}$ pathway in UE7T-13 cells (34) and that the expression of VCAM-1 in high cell density culture was significantly inhibited by treatment with IKK-2 inhibitor VI. In the present study, we investigated the effects of RTK inhibitors on VCAM-1 expression. As illustrated in Fig. 1, the high cell density-induced VCAM-1 expression decreased in a dosedependent manner upon the addition of PDGFR inhibitors (SU11652 and PDGFR tyrosine kinase inhibitor IV) to the cell culture. However, the addition of the following RTK inhibitors did not decrease VCAM-1 expression: vascular endothelial growth factor receptor (VEGFR) inhibitors (SU1498 and VEGFR kinase inhibitor I), an insulin-like growth factor-1 receptor (IGF-1R) inhibitor (AG1024), an epidermal growth factor receptor (EGFR) inhibitor (AG1478), fibroblast growth factor receptor (FGFR) inhibitors (SU4984 and SU5402), and a colony stimulating factor-1 receptor (CSF1R) tyrosine kinase inhibitor (Fig. 1A). Similarly, the addition of a receptor type serine/threonine kinase inhibitor, such as a transforming growth factor- $\beta$ receptor (TGF $\beta R$ ) inhibitor (SB431542) did not decrease VCAM-1 expression (Fig. 1A).

Role of PDGFR $\beta$ on high cell density-induced VCAM-1 expression. In order to determine the role of PDGFRs in the high cell density-induced intracellular signal transduction culminating into VCAM-1 expression in UE7T-13 cells, we investigated the effects of knocking down PDGFR $\alpha$ and PDGFR $\beta$ expression on VCAM- 1 expression. As shown in Fig. 2, VCAM-1 mRNA expression in high cell density culture was reduced effectively by transfection with siRNA targeting PDGFR $\beta$, but not siRNA targeting PDGFR $\alpha$. In order to investigate whether PDGFR $\beta$ mediates the intracellular signal for VCAM-1 expression in a ligand-dependent manner, PDGF-BB was added to the UE7T-13 cell culture at high and low cell densities. As shown in Fig. 3A, PDGF-BB did not affect the level of VCAM-1 mRNA expression in the MSCs at low or high cell densities. By contrast, exogenously added recombinant human PDGF-BB markedly accelerated the proliferation of UE7T-13 cells, suggesting that functional PDGFR is present at the cell surface in UE7T-13 cells (Fig. 3B).

Role of N-cadherin in high cell density-induced VCAM-1 expression. A previous study revealed that a mechanostressinduced intracellular signal is mediated by an RTK in ECs, even if the receptor was not stimulated with any ligand: vascular endothelial (VE)-cadherin forms a mechanosensory complex with VEGFR2 in ECs in a ligand-independent manner (37). Therefore, in this study, we investigated the role of N-cadherin as a major structural molecule in AJs in MSCs on high cell density-induced VCAM-1 expression. UE7T-13 cells were transfected with vectors expressing either full-length $\mathrm{N}$-cadherin or a truncated version lacking the intracellular domain. The expression of $\mathrm{N}$-cadherin, which localizes on the

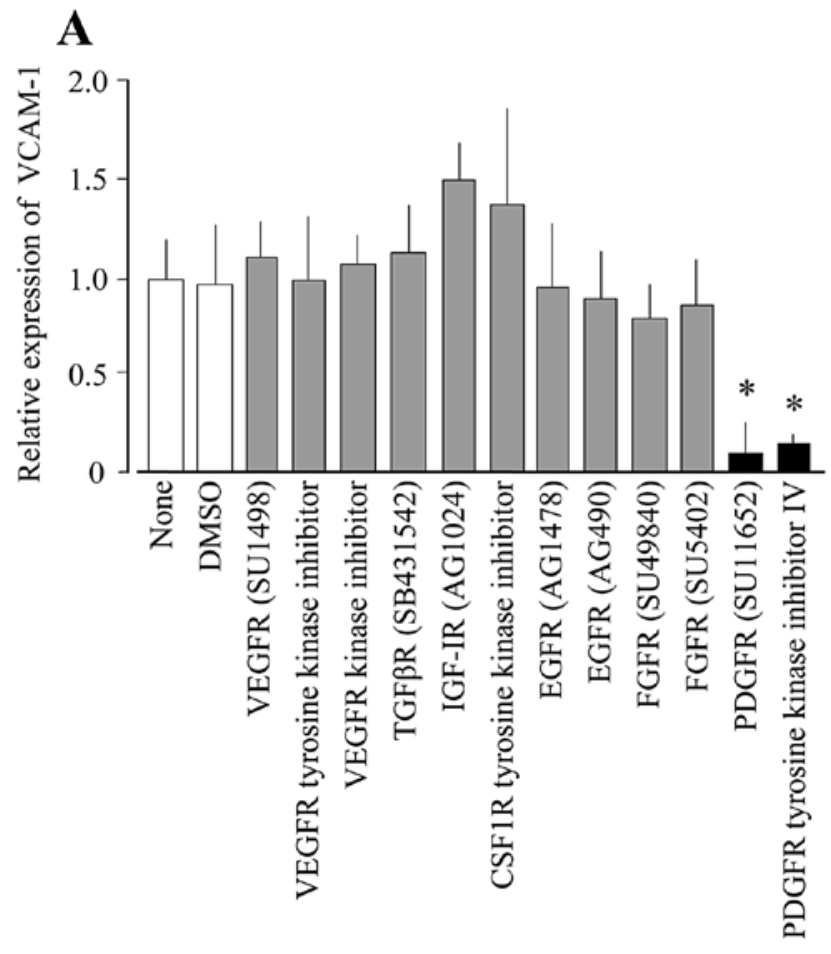

\section{B}

SU11652

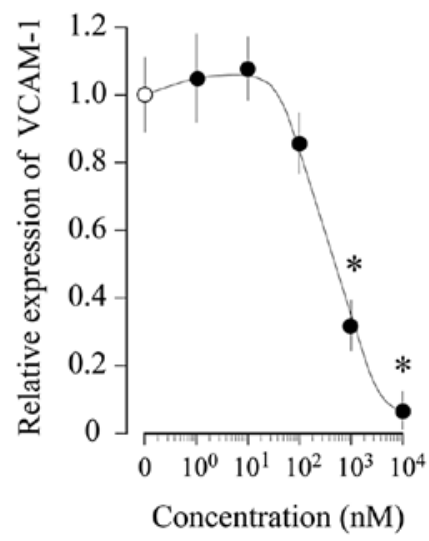

PDGF receptor tyrosine

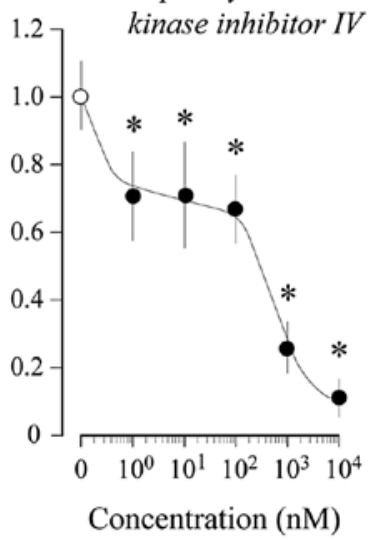

Figure 1. High cell density-induced VCAM-1, vascular cell adhesion molecule-1 (VCAM-1) expression was decreased by platelet-derived growth factor receptor (PDGFR) tyrosine kinase inhibitors. (A) UE7T-13 cells were seeded on 24-well dish in high-cell density condition. After $6 \mathrm{~h}$ of culture, the cells were treated with $10 \mu \mathrm{M}$ of various receptor type protein kinase inhibitors, and then maintained for $18 \mathrm{~h}$. Relative mRNA expression levels of VCAM-1 were analyzed by qRT-PCR. (B) The cells were treated with the PDGFR tyrosine kinase specific inhibitors (SU11652 and PDGFR tyrosine kinase inhibitor IV) at various doses as indicated for $18 \mathrm{~h}$. Relative mRNA expression levels of VCAM-1 were analyzed by qRT-PCR. Data represent the means of 3 individual experiments $(\mathrm{n}) \pm \mathrm{SD}$. "p $<0.05$, statistically significant vs. untreated control.

cell surface, was confirmed by flow cytometry (Fig. 4A), and then the cells were seeded at a high density. Under conditions of high density, the overexpression of full-length $\mathrm{N}$-cadherin markedly increased the level of VCAM-1 mRNA expression. By contrast, the overexpression of the truncated $\mathrm{N}$-cadherin markedly reduced the high cell density-induced VCAM-1 expression (Fig. 4B). These results indicate that, in MSCs, the 

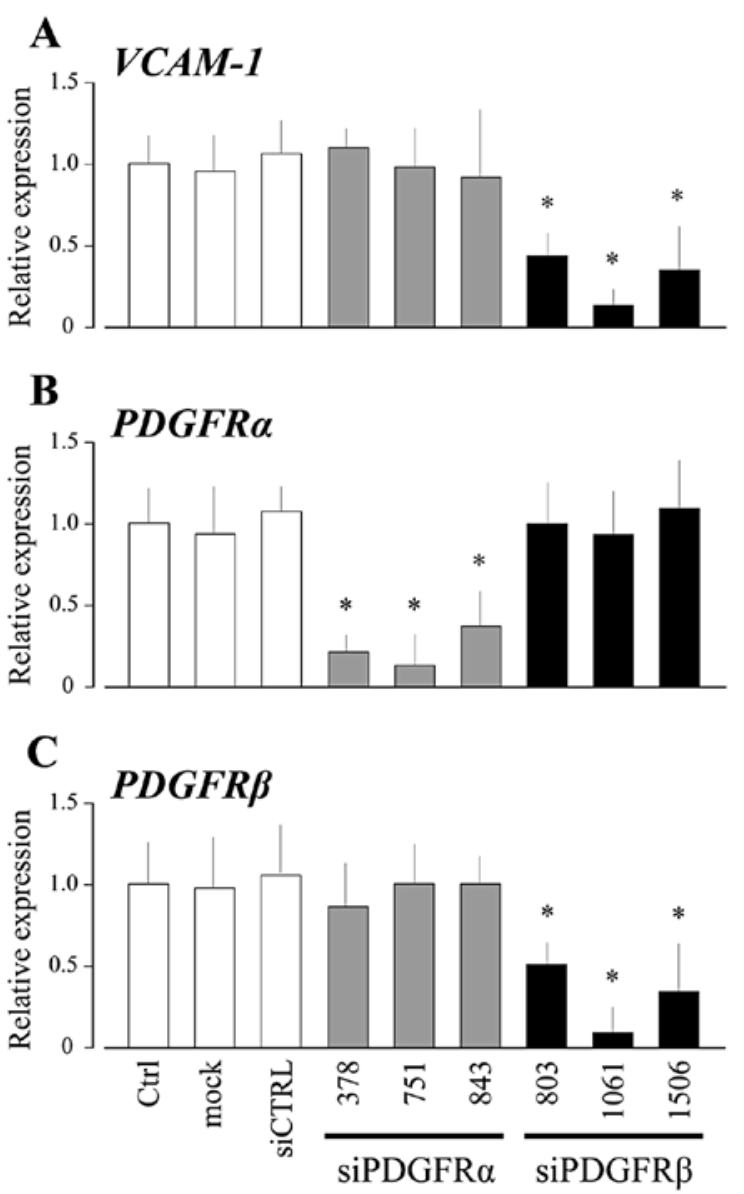

Figure 2. High cell density-induced VCAM-1, vascular cell adhesion molecule-1 (VCAM-1) expression was reduced by siRNA-mediated plateletderived growth factor receptor (PDGFR) knockdown. UE7T-13 cells were transfected with non-targeting control (siCTRL), PDGFR $\alpha$-specific siRNA (siPDGFR $\alpha$ ) and PDGFR $\beta$-specific siRNA (siPDGFR $\beta$ ). Following siRNA transfection, the cells were replated on plastic culture plates and maintained at a high-cell density condition for $24 \mathrm{~h}$. Relative mRNA expression levels of (A) VCAM-1, (B) PDGFR $\alpha$ and (C) PDGFR $\beta$ were analyzed by qRT-PCR. Data represent the means of 3 individual experiments (n) \pm SD. " $\mathrm{p}<0.05$, statistically significant vs. untransfected control (Ctrl).

high cell density induction of VCAM-1 expression is mediated by $\mathrm{N}$-cadherin.

Signaling pathway for high cell density-induced VCAM-1 expression. In ECs, even if VEGFR2 is not stimulated with VEGF, the VE-cadherin-VEGFR2 complex responds to a subset of endothelial shear stresses, resulting in the activation of NF-кB-mediated Src signaling that upregulates VCAM-1 expression (37). Therefore, in this study, we investigated the phosphorylation status of Src by western blot analysis. As shown in Fig. 5, the phosphorylation levels of Src in UE7T-13 cells were markedly upregulated by the overexpression of full-length $\mathrm{N}$-cadherin (pCDH2-full). On the other hand, the overexpression of the truncated mutant (pCDH2- $\Delta$ ) had no effect compared with the control vector-transfected cells (pCTRL). This result strongly suggests that the phosphorylation of Src through the intracellular domain of $\mathrm{N}$-cadherin plays an important role in the regulation of VCAM-1 expression by cell-cell adhesion.
$\mathbf{A}$
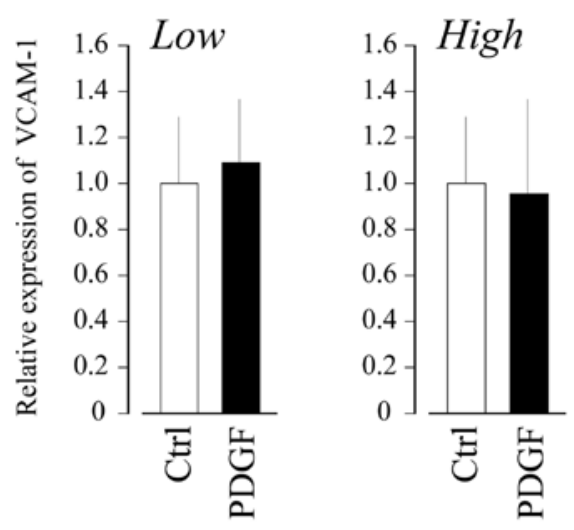

\section{B}

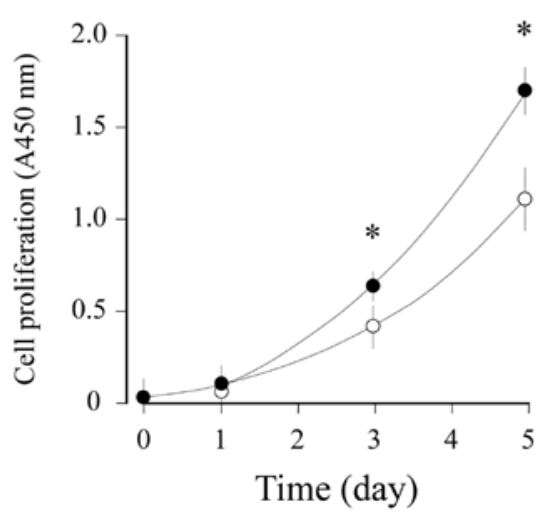

Figure 3. Platelet-derived growth factor (PDGF) does not affect high cell density-induced vascular cell adhesion molecule-1 (VCAM-1) expression. (A) UE7T-13 cells were seeded at low- or high-cell densities, and maintained for $6 \mathrm{~h}$. The cells were then treated with $10 \mathrm{ng} / \mathrm{ml}$ of recombinant human PDGF-BB for $18 \mathrm{~h}$. VCAM-1 mRNA expression levels were then analyzed by qRT-PCR. (B) UE7T-13 cells were seeded on 96-well plates, then maintained for 1,3 and 5 days under a treatment with (closed circles) or without (open circles) $10 \mathrm{ng} / \mathrm{ml}$ of recombinant human PDGF-BB. Cell proliferation activity was analyzed using the WST-1 assay reagent by measurement of absorbance at $450 \mathrm{~nm}$. ${ }^{*} \mathrm{p}<0.05$, statistically significant vs. untreated control. Data represent the means of 3 individual experiments $(n) \pm S D$.

\section{Discussion}

VCAM-1 is an important marker for enriching migratory, multipotent and proliferative cells from culture-expanded MSCs (11). In addition, it has been shown that VCAM-1 is upregulated in bone marrow-derived MSCs cultured to overconfluency (38). However, a comprehensive analysis of the types of adhesion molecules that play important roles in cell-cell contact between MSCs has not been performed. In a recent study, we demonstrated that VCAM-1 expression was markedly upregulated in human bone marrow-derived MSCs at high cell density through the activation of the NF- $\mathrm{KB}$ pathway (34).

Notably, a previous study revealed that a mechanostressinduced intracellular signal is mediated by an RTK in ECs in a ligand-independent manner: VE-cadherin forms a mechanosensory complex with VEGFR2 in ECs that responds to a subset of endothelial shear stresses, resulting in the activation of NF- $\mathrm{KB}$-mediated Src signaling, upregulating 

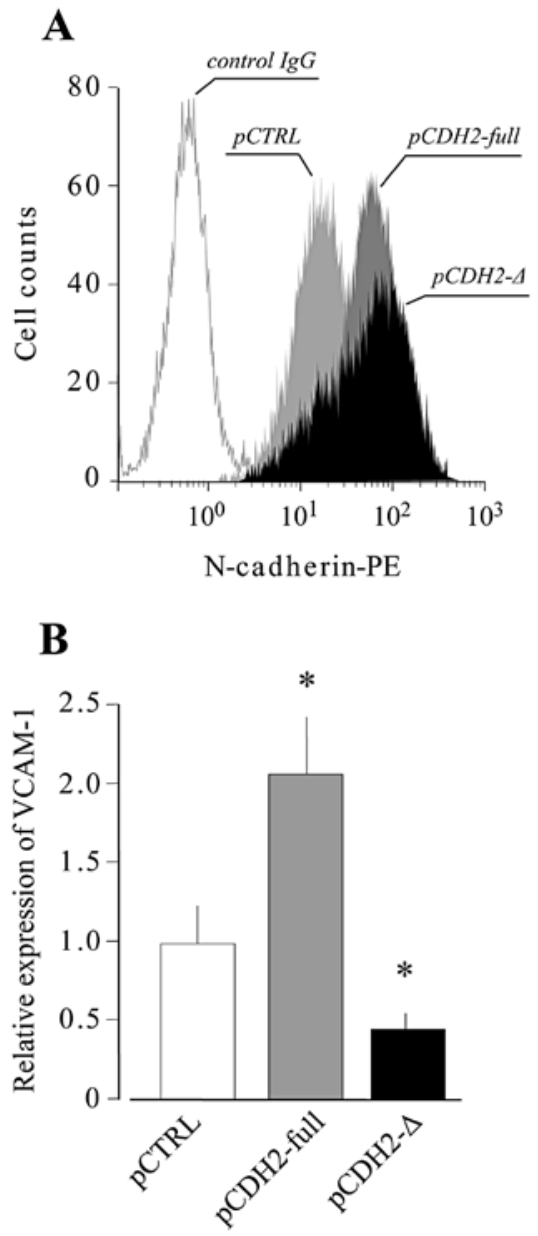

Figure 4. Deletion of the intracellular domain of N-cadherin decreased the high cell density-induced vascular cell adhesion molecule-1 (VCAM-1) expression. (A) $\mathrm{N}$-cadherin expression vectors ( $\mathrm{pCDH} 2$-full or $\mathrm{pCDH} 2-\Delta$ ) were transfected into the UE7T-13 cells. After $48 \mathrm{~h}$, the cells were stripped and suspended in PBS containing $0.5 \%$ FBS and $2 \mathrm{mM}$ EDTA. The cells $\left(1.0 \times 10^{5}\right)$ were incubated with anti-N-cadherin antibody, and then incubated with PE-conjugated secondary antibody. The acquisition was performed in EPICS XL ADC System. (B) UE7T-13 cells were transfected with control (pCTRL), pCDH2full, or pCDH2- $\Delta$ for $48 \mathrm{~h}$. Following transfection, the cells were replaced on plastic culture plates and maintained at a high cell density condition for $24 \mathrm{~h}$. Relative mRNA expression levels of VCAM-1 were analyzed by qRT-PCR Data represent the means of 3 individual experiments (n) \pm SD. * p $<0.05$, statistically significant vs. control vector transfection (pCTRL).

VCAM-1 expression, even when VEGFR2 is not stimulated with VEGF (37). Therefore, we hypothesized that an RTK may similarly mediate the cell-cell contact-induced signal responsible for upregulating VCAM-1 expression in MSCs. When we evaluated the effects of various RTK inhibitors on high cell density-induced VCAM-1 expression, we found that VCAM-1 expression was decreased by PDGFR inhibitors (SU11652 and PDGFR tyrosine kinase inhibitor IV) in a dosedependent manner (Fig. 1). However, other RTK inhibitors, e.g., inhibitors of VEGFR, IGF1R, EGFR and FGFR, as well as a receptor type serine/threonine kinase TGF $\beta \mathrm{R}$ inhibitor, did not decrease VCAM-1 expression (Fig. 1A). In general, PDGFR is activated by PDGF as its ligand. PDGF has four isoforms (A-D) that form homo- or heterodimers, such as PDGF-AA, PDGF-AB and PDGF-BB (39). Of these, PDGF-BB exhibits the strongest activity (39) and has been approved by the FDA
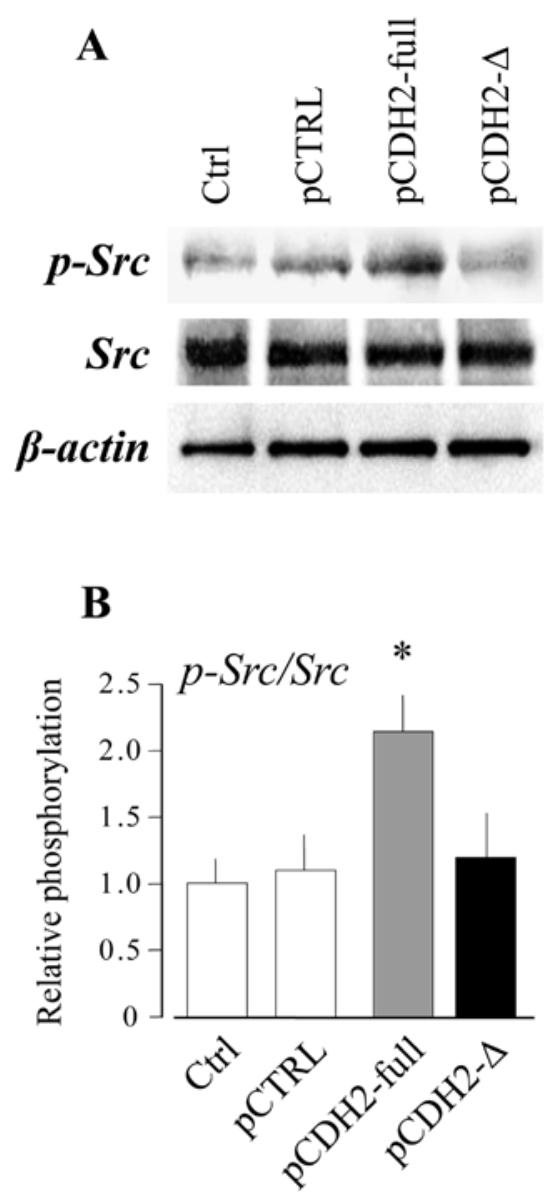

Figure 5. Deletion of the intracellular domain of N-cadherin suppresses phosphorylation of Src. (A) N-cadherin expression vectors (pCDH2-full or pCDH2- $\Delta$ ) were transfected with UE7T-13 cells for $48 \mathrm{~h}$. The cells were then placed on plastic culture plates and maintained at a high cell density condition for $24 \mathrm{~h}$. The cells were lysed in RIPA buffer. Each sample containing equal amounts of protein was separated by SDS-PAGE and transferred onto a PVDF membrane. The membrane was incubated with a primary anti-Src (Src), anti-phospho-Src (p-Src) and anti- $\beta$-actin (ACTB) antibody as a loading control. The blots were then incubated with HRP conjugated secondary antibody and developed using the ECL Western Blotting Analysis System. (B) Densitometric analysis was performed using ImageJ software. Data are expressed as the ratio of the phosphorylated to total molecular bands. Data represent the means of 3 individual experiments $(n) \pm S D$. " $p<0.05$, statistically significant vs. untransfected cells (Ctrl).

for the treatment of patients with bone defects in the oral and maxillofacial regions (40-43). PDGF-BB is mainly produced by platelets and has been implicated in tissue repair (fracture repair) (39). PDGFR has two isoforms ( $\alpha$ and $\beta$ ) that also form homo- or heterodimers, such as PDGFR $\alpha / \alpha, \alpha / \beta$ and $\beta / \beta$ (39). PDGFR $\alpha$ is reportedly expressed in MSCs and osteoblast progenitor cells, and PDGFR $\alpha$-positive cells exhibit a high osteoblastic differentiation capacity (44). In MSCs, a recent study provided evidence that PDGF-BB promotes PDGF $\alpha$ positive cell migration into artificial bones without inhibiting osteoblastogenesis (45). In this study, high cell density-induced VCAM-1 expression was significantly suppressed by the knockdown of PDGFR $\beta$ (Fig. 2). Of note, the PDGF-BBinduced PDGFR activation did not affect the level of VCAM-1 mRNA expression in MSCs at both low and high cell densities (Fig. 3A), although PDGF-BB clearly upregulated MSC 
proliferation (Fig. 3B). These results strongly suggest that the high cell density-induced intracellular signal to upregulate VCAM-1 expression in MSCs was mediated by PDGFR $\beta$ in a PDGF-BB-independent manner.

In MSCs, VE-cadherin does not exhibit dominant expression, although $\mathrm{N}$-cadherin does (46). Therefore, it may be possible that the formation of a mechanosensory interaction occurs between $\mathrm{N}$-cadherin and PDGFR $\beta$ in high cell density cultures and that this interaction may then activate the NF- $\mathrm{NB}$-mediated signaling pathway, thus upregulating VCAM-1 expression. In support of this hypothesis, in the present study, the overexpression of full-length $\mathrm{N}$-cadherin in UE7T-13 cells markedly increased high cell density-induced VCAM-1 expression, whereas the overexpression of truncated $\mathrm{N}$-cadherin lacking its intracellular domain suppressed the high cell density-induced VCAM-1 expression (Fig. 4). As, in ECs, the VE-cadherin-VEGFR2 complex responds to a subset of endothelial shear stresses by activating NF- $\kappa \mathrm{B}$-mediated Src signaling (37). Therefore, we examined the phosphorylation status of Src by western blot analysis and found that the phosphorylation levels of Src in UE7T-13 cells were enhanced at high cell density (Fig. 5A). Src phosphorylation levels in the UE7T-13 cells were also upregulated by the overexpression of full-length N-cadherin (Fig. 5B). By contrast, the overexpression of the truncated mutant did not affect Src phosphorylation. In addition, in a previous study, we demonstrated that high cell density-induced VCAM-1 expression was decreased by the Src inhibitor, PP2 analogue (34). These results strongly suggest that the phosphorylation of Src through the intracellular domain of $\mathrm{N}$-cadherin plays an important role in the upregulation of VCAM-1 by cell-cell adhesion.

In conclusion, the data presented in our study demonstrate that cell-cell adhesion induced by high cell density through $\mathrm{N}$-cadherin enhances the expression of VCAM-1 via PDGFR $\beta$ in a ligand-independent manner in human bone marrowderived MSCs. These findings may eventually lead to the development of new, MSC-based clinical therapies in regenerative medicine.

\section{Acknowledgements}

This study was supported in part by JSPS KAKENHI Grant nos. 25463053 to N.C., 23592896 to A.I.; the Open Research Project from the Ministry of Education, Culture, Sports, Science and Technology of Japan, 2008-2012; and Grant-in-Aid for Strategic Medical Science Research Centre from the Ministry of Education, Culture, Sports, Science and Technology of Japan, 2010-2014.

\section{References}

1. Prockop DJ: Marrow stromal cells as stem cells for nonhematopoietic tissues. Science 276: 71-74, 1997.

2. Aslan H,Zilberman Y, Kandel L, et al: Osteogenic differentiation of noncultured immunoisolated bone marrow-derived CD105 cells. Stem Cells 7: 1728-1737, 2006.

3. Battula VL, Treml S, Bareiss PM, et al: Isolation of functionally distinct mesenchymal stem cell subsets using antibodies against CD56, CD271, and mesenchymal stem cell antigen-1. Haematologica 94: 173-184, 2009.

4. Boiret N, Rapatel C, Veyrat-Masson R, et al: Characterization of nonexpanded mesenchymal progenitor cells from normal adult human bone marrow. Exp Hematol 33: 219-225, 2005.
5. Bühring HJ, Battula VL, Treml S, et al: Novel markers for the prospective isolation of human MSC. Ann NY Acad Sci 1106: 262-271, 2007.

6. Gronthos S, Zannettino AC, Hay SJ, et al: Molecular and cellular characterisation of highly purified stromal stem cells derived from human bone marrow. J Cell Sci 116: 1827-1835, 2003.

7. Quirici N, Soligo D, Bossolasco P, et al: Isolation of bone marrow mesenchymal stem cells by anti-nerve growth factor receptor antibodies. Exp Hematol 30: 783-791, 2002.

8. Sacchetti B, Funari A, Michienzi S, et al: Self-renewing osteoprogenitors in bone marrow sinusoids can organize a hematopoietic microenvironment. Cell 131: 324-336, 2007.

9. Honczarenko M, Le Y, Swierkowski M, et al: Human bone marrow stromal cells express a distinct set of biologically functional chemokine receptors. Stem Cells 24: 1030-1041, 2006.

10. Wagner W, Horn P, Castoldi M, et al: Replicative senescence of mesenchymal stem cells: a continuous and organized process. PLoS One 3: e2213, 2008.

11. Mabuchi Y, Morikawa S, Harada S, et al: LNGFR(+)THY-1(+) VCAM-1(hi+) cells reveal functionally distinct subpopulations in mesenchymal stem cells. Stem Cell Reports 1: 152-165, 2013.

12. Deschaseaux F, Gindraux F, Saadi R, et al: Direct selection of human bone marrow mesenchymal stem cells using an antiCD49a antibody reveals their CD45med, low phenotype. Br J Haematol 122: 506-517, 2003.

13. Jones EA, English A, Kinsey SE, et al: Optimization of a flow cytometry-based protocol for detection and phenotypic characterization of multipotent mesenchymal stromal cells from human bone marrow. Cytometry B Clin Cytom 70: 391-399, 2006.

14. Matsuyoshi $\mathrm{N}$ and Imamura S: Multiple cadherins are expressed in human fibroblasts. Biochem Biophys Res Commun 235: 355-358, 1997.

15. Simonneau L, Kitagawa M, Suzuki S and Thiery JP: Cadherin 11 expression marks the mesenchymal phenotype: towards new functions for cadherins? Cell Adhes Commun 3: 115-130, 1995.

16. Hatta $\mathrm{K}$ and Takeichi M: Expression of N-cadherin adhesion molecules associated with early morphogenetic events in chick development. Nature 320: 447-449, 1986.

17. Akitaya $\mathrm{T}$ and Bronner-Fraser M: Expression of cell adhesion molecules during initiation and cessation of neural crest cell migration. Dev Dyn 194: 12-20, 1992.

18. De Wever O, Westbroek W, Verloes A, et al: Critical role of $\mathrm{N}$-cadherin in myofibroblast invasion and migration in vitro stimulated by colon-cancer-cell-derived TGF-beta or wounding. J Cell Sci 117: 4691-4703, 2004.

19. Kashima T, Nakamura K, Kawaguchi J, et al: Overexpression of cadherins suppresses pulmonary metastasis of osteosarcoma in vivo. Int J Cancer 104: 147-154, 2003.

20. Radice GL, Rayburn H, Matsunami H, et al: Developmental defects in mouse embryos lacking N-cadherin. Dev Biol 181: 64-78, 1997.

21. García-Castro MI, Vielmetter E and Bronner-Fraser M: $\mathrm{N}$-Cadherin, a cell adhesion molecule involved in establishment of embryonic left-right asymmetry. Science 288: 1047-1051, 2000.

22. Hinz B, Pittet P, Smith-Clerc J, et al: Myofibroblast development is characterized by specific cell-cell adherens junctions. Mol Biol Cell 15: 4310-4320, 2004

23. Tomasek JJ, Gabbiani G, Hinz B, et al: Myofibroblasts and mechano-regulation of connective tissue remodelling. Nat Rev Mol Cell Biol 3: 349-363, 2002.

24. Marthiens V, Gavard J, Lambert M and Mège RM: Cadherin-based cell adhesion in neuromuscular development. Biol Cell 94: 315-326, 2002.

25. Krauss RS, Cole F, Gaio U, et al: Close encounters: regulation of vertebrate skeletal myogenesis by cell-cell contact. J Cell Sci 118: 2355-2362, 2005

26. Marie PJ: Role of N-cadherin in bone formation. J Cell Physiol 190: 297-305, 2002.

27. Ahrens PB, Solursh M and Reiter RS: Stage-related capacity for limb chondrogenesis in cell culture. Dev Biol 60: 69-82, 1977.

28. San Antonio JD and Tuan RS: Chondrogenesis of limb bud mesenchyme in vitro: stimulation by cations. Dev Biol 115: 313-324, 1986.

29. Oberlender SA and Tuan RS: Spatiotemporal profile of $\mathrm{N}$-cadherin expression in the developing limb mesenchyme. Cell Adhes Commun 2: 521-537, 1994.

30. Tavella S, Raffo P, Tacchetti C, et al: N-CAM and N-cadherin expression during in vitro chondrogenesis. Exp Cell Res 215: 354-362, 1994. 
31. DeLise AM and Tuan RS: Alterations in the spatiotemporal expression pattern and function of $\mathrm{N}$-cadherin inhibit cellular condensation and chondrogenesis of limb mesenchymal cells in vitro. J Cell Biochem 87: 342-359, 2002.

32. Delise AM and Tuan RS: Analysis of N-cadherin function in limb mesenchymal chondrogenesis in vitro. Dev Dyn 225: 195-204, 2002.

33. Tuan RS: Cellular signaling in developmental chondrogenesis: N-cadherin, Wnts, and BMP-2. J Bone Joint Surg Am 85: 137-141, 2003

34. Nishihira S, Okubo N, Takahashi N, et al: High-cell densityinduced VCAM-1 expression inhibits the migratory ability of mesenchymal stem cells. Cell Biol Int 35: 475-481, 2011.

35. Mori T, Kiyono T, Imabayashi H, et al: Combination of hTERT and bmi-1, E6, or E7 induces prolongation of the life span of bone marrow stromal cells from an elderly donor without affecting their neurogenic potential. Mol Cell Biol 25: 5183-5195, 2005.

36. Shimomura T, Yoshida Y, Sakabe T, et al: Hepatic differentiation of human bone marrow-derived UE7T-13 cells: Effects of cytokines and CCN family gene expression. Hepatol Res 37: 1068-1079, 2007.

37. Liu Y, Sweet DT, Irani-Tehrani M, et al: Shc coordinates signals from intercellular junctions and integrins to regulate flowinduced inflammation. J Cell Biol 182: 185-196, 2008.

38. Lee RH, Seo MJ, Pulin AA, et al: The CD34-like protein PODXL and alpha6-integrin (CD49f) identify early progenitor MSCs with increased clonogenicity and migration to infarcted heart in mice. Blood 113: 816-826, 2009.

39. Hollinger JO, Hart CE, Hirsch SN, et al: Recombinant human platelet-derived growth factor: biology and clinical applications. J Bone Joint Surg Am 90: 48-54, 2008.
40. Jayakumar A, Rajababu P, Rohini S, et al: Multi-centre, randomized clinical trial on the efficacy and safety of recombinant human platelet-derived growth factor with $\beta$-tricalcium phosphate in human intra-osseous periodontal defects. J Clin Periodontol 38: 163-172, 2011.

41. Ridgway HK, Mellonig JT and Cochran DL: Human histologic and clinical evaluation of recombinant human platelet-derived growth factor and beta-tricalcium phosphate for the treatment of periodontal intraosseous defects. Int J Periodontics Restorative Dent 28: 171-179, 2008.

42. Nevins M, Giannobile WV, McGuire MK, et al: Platelet-derived growth factor stimulates bone fill and rate of attachment level gain: results of a large multicenter randomized controlled trial. J Periodontol 76: 2205-2215, 2005.

43. McGuire MK, Kao RT, Nevins M and Lynch SE: rhPDGF-BB promotes healing of periodontal defects: 24-month clinical and radiographic observations. Int J Periodontics Restorative Dent 26: 223-231, 2006.

44. Morikawa S, Mabuchi Y, Kubota Y, et al: Prospective identification, isolation, and systemic transplantation of multipotent mesenchymal stem cells in murine bone marrow. J Exp Med 206: 2483-2496, 2009.

45. Yoshida S, Iwasaki R, Kawana $\mathrm{H}$, et al: PDGFBB promotes PDGFR $\alpha$-positive cell migration into artificial bone in vivo. Biochem Biophys Res Commun 421: 785-789, 2012.

46. Wuchter P, Boda-Heggemann J, Straub BK, et al: Processus and recessus adhaerentes: giant adherens cell junction systems connect and attract human mesenchymal stem cells. Cell Tissue Res 328: 499-514, 2007. 